\title{
Star-formation efficiency in the outer Galaxy
}

\section{Natsuko Izumi ${ }^{1}$, Naoto Kobayashi ${ }^{2}$, Chikako Yasui ${ }^{1}$, Alan T. Tokunaga $^{3}$, Masao Saito ${ }^{4,5}$ and Satoshi Hamano ${ }^{6}$}

\author{
${ }^{1}$ National Astronomical Observatory of Japan, 2-21-1, Osawa, Mitaka, Tokyo, 181-8588, Japan \\ email: natsuko.izumi@nao.ac.jp \\ ${ }^{2}$ Institute of Astronomy, School of Science, University of Tokyo, \\ 2-21-1, Osawa, Mitaka, Tokyo 181-0015, Japan \\ ${ }^{3}$ Institute for Astronomy, University of Hawaii, 2680 Woodlawn Drive, \\ Honolulu, HI 96822, USA \\ ${ }^{4}$ Nobeyama Radio Observatory, 462-2 Nobeyama, Minamimaki-mura, \\ Minamisaku-gun, Nagano 384-1305, Japan \\ ${ }^{5}$ The Graduate University of Advanced Studies, (SOKENDAI), \\ 2-21-1 Osawa, Mitaka, Tokyo 181-8588, Japan \\ ${ }^{6}$ Laboratory of Infrared High-resolution spectroscopy (LiH), Koyama Astronomical \\ Observatory, Kyoto Sangyo University, \\ Motoyama, Kamigamo, Kita-ku, Kyoto 603-8555, Japan
}

\begin{abstract}
We report the results of new survey of star-forming regions in the outer Galaxy at Galactocentric radius of more than $13.5 \mathrm{kpc}$, where the environment is significantly different from that in the solar neighborhood.
\end{abstract}

Keywords. stars: formation, infrared:stars, ISM:clouds

\section{Introduction}

The environment of the outer Galaxy is significantly different from the environment around the solar neighbourhood with lower gas density (e.g., Wolfire et al. 2003) and lower metallicity (e.g., Smartt \& Rolleston 1997). Therefore, the outer Galaxy serves as an excellent laboratory for studying the star-forming processes in an environment with characteristics similar to dwarf galaxies as well as the early phase of the formation of our Galaxy (Ferguson et al. 1998; Kobayashi et al. 2008). It is known that the star-formation rate (SFR) or constant star-formation efficiency (cSFE), for converting HI gas to stars, decreases significantly at under low surface gas density (e.g., Kennicutt \& Evans 2012). A similar trend is also observed in our Galaxy up to Galactocentric radius $\left(R_{\mathrm{G}}\right)$ of about $15 \mathrm{kpc}$ (Kennicutt \& Evans 2012). Both SFR and cSFE start decreasing significantly at $R_{\mathrm{G}} \sim 13.5 \mathrm{kpc}$, then decrease to about $1 / 8$ and $1 / 4$ of that of the solar neighborhood, respectively, at $R_{\mathrm{G}} \sim 15 \mathrm{kpc}$. Thus, in the outer Galaxy, we may examine the causes of such trends in much greater detail than in extra-galactic objects. This study is a part of Izumi (2016).

\section{New survey of star-forming regions in the outer Galaxy}

To clarify the global nature of star-formation activity in the outer Galaxy, we need a statistical sample of star-forming regions. However, the outer Galaxy has never been comprehensively surveyed because of lack of infrared (IR) survey data deep enough for the large distances, and only about 30 star-forming regions are known so far (e.g., Snell et al. 2002; Brand \& Wouterloot, 2007). Therefore, we have examined the Wide-Field Infrared 


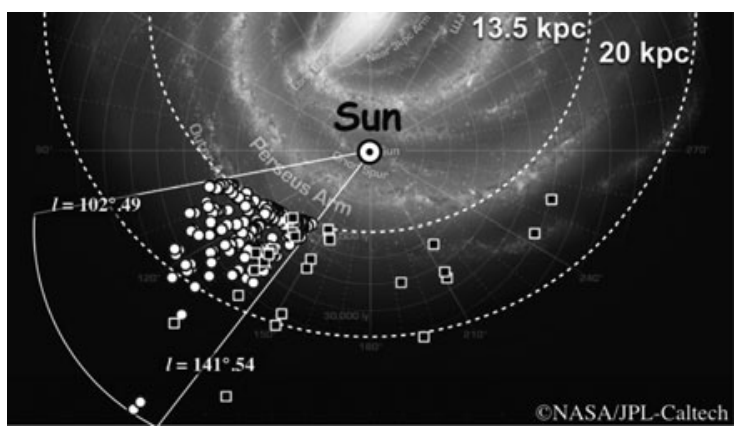

Figure 1. Distribution of star-forming regions in the second and third quadrants (adapted from the image of Milky Way Galaxy from NASA's Spitzer Space Telescope by NASA/JPL-Caltech). The white circles and black squares show newly identified star-forming regions and known starforming regions, respectively. The white fan-shaped region shows the survey area of FCRAO CO outer Galaxy survey $\left(102^{\circ} .49 \leqslant l \leqslant 141^{\circ} .54,-3^{\circ} .03 \leqslant b \leqslant 5^{\circ} .41\right)$, and thus also that of the present survey.

Survey Explorer (WISE) mid infrared (MIR) all sky survey data (Wright et al. 2010), which has a high potential for detecting distant star-forming regions in the outer Galaxy. WISE has achieved a great increase in sensitivity, and is about a hundred times more sensitive than IRAS (Wright et al. 2010). We developed a simple criteria for identifying star-forming regions with WISE colors, which is effective when used with available CO survey data in the outer Galaxy. We employed FCRAO CO outer Galaxy survey data (Heyer et al. 1998; Brunt et al. 2003) to search for star-forming regions within an area of $320 \mathrm{deg}^{2}$. As a result, we identified 711 new candidate of star-forming regions (Figure 1), which enables a statistical study of star-formation activity in the outer Galaxy for the first time.

\section{Spiral distribution of star-forming regions in the outer Galaxy}

From the distribution of newly identified star-forming regions, we found a gap in the distribution at $R_{\mathrm{G}} \sim 15 \mathrm{kpc}$ between two separate groups at $R_{\mathrm{G}} \sim 14 \mathrm{kpc}$ and $R_{\mathrm{G}}=16-$ $17 \mathrm{kpc}$. The former group is likely to be associated with the outer arm at $R_{\mathrm{G}} \sim 14 \mathrm{kpc}$ in the direction of the anticenter (e.g., Hachisuka et al. 2015) and the latter group appears to be a part of a new arm beyond the outer arm. This new arm has slightly smaller $R_{\mathrm{G}}$ than that of the newly suggested arm by Strasser et al. (2007) and Sun et al. (2015). Sun et al. (2015) proposed that their new arm is connected to the Scutum-Centaurus arm. While our data shows a number of star-forming regions on the trail of Sun et al. (2015)'s proposed new arm, our new arm appears to form another branch from the outer arm. Obviously, surveys of star-forming regions at $l=60 \sim 100^{\circ}$ will be very important for understanding these arm structures.

\section{Star-formation efficiency in the outer Galaxy}

Using the newly identified star-forming regions, we investigate SFE of each molecular cloud as opposed to global SFE used for constructing the Kennicutt-Schmidt law. We developed two empirical SFE-indices of molecular cloud, 1) the number ratio of clouds with star-forming region to all clouds, and 2) MIR luminosities per cloud mass, to examine the variation of SFE with $R_{\mathrm{G}}$. Although star-formation processes are predicted to change with environment, we could not find any clear trend in the range $R_{\mathrm{G}}=13.5-20 \mathrm{kpc}$. For 


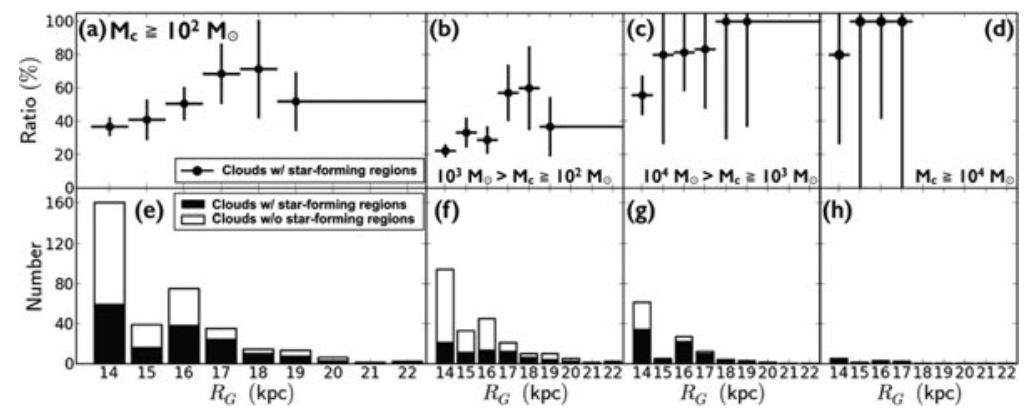

Figure 2. (a): Galactocentric variation of the ratio of clouds w/star-forming regions to the total number of clouds with cloud mass $\left(M_{\mathrm{c}}\right)$ of larger than $10^{2} M_{\odot}$ (SFE-index 1). (b) (d): Same as (a) plot but using clouds in three $M_{\mathrm{c}}$ ranges (b: $10^{3} M_{\odot}>M_{\mathrm{c}} \geqslant 10^{2} M_{\odot}$, c: $10^{4} M_{\odot}>M_{\mathrm{c}}$ $\left.\geqslant 10^{3} M_{\odot}, \mathrm{d}: M_{\mathrm{c}} \geqslant 10^{4} M_{\odot}\right)$. (e): Galactocentric variation of the number of clouds with $M_{\mathrm{c}} \geqslant$ $10^{2} M_{\odot}$. The black and white bars show the number of clouds w/ and w/o star-forming regions, respectively. (f) $\sim(\mathrm{h})$ : Same as (e) plot but using clouds in three $M_{\mathrm{c}}$ ranges (f: $10^{3} M_{\odot}>M_{\mathrm{c}} \geqslant$ $\left.10^{2} M_{\odot}, \mathrm{g}: 10^{4} M_{\odot}>M_{\mathrm{c}} \geqslant 10^{3} M_{\odot}, \mathrm{h}: M_{\mathrm{c}} \geqslant 10^{4} M_{\odot}\right)$.

example, Figure 2 shows the variation of SFE-index 1 with $R_{\mathrm{G}}$. Although the index may appear to increase slightly with increasing $R_{\mathrm{G}}$ (in other words, decreasing gas density and metallicity), we could only safely say that the ratio does not decrease in view of the statistics at larger $R_{\mathrm{G}}$. We note that such increasing trend is not shown in the variation of SFE-index 2 with $R_{\mathrm{G}}$. This suggests that the star-formation processes inside molecular cloud do not heavily depend on the environmental parameters, such as metallicity. If this is the case, low SFE found in the outer regions of disk galaxies (e.g., Bigiel et al. 2010) simply reflects the smaller number of molecular clouds in such region. SFE in the inner regions of galaxies, where the interstellar medium (ISM) is dominated by $\mathrm{H}_{2}$ molecular gas, is suggested to depend only on the amount of $\mathrm{H}_{2}$ gas, but not that of HI gas (e.g., Schruba et al. 2011). The above results suggest that SFE in the outer Galaxy, where the ISM is dominated by HI gas, also depends simply on the amount of $\mathrm{H}_{2}$ gas as in the inner region of galaxies.z We plan to expand the survey area to detect larger number of samples, in particular, at $R_{\mathrm{G}} \geqslant 17 \mathrm{kpc}$ for improving the statistical reliability of our study in the near future.

\section{References}

Bigiel, F., Leroy, A., Walter, F., et al. 2010, AJ, 140, 1194

Brand, J. \& Wouterloot, J. G. A. 2007, A\&A, 464, 909

Brunt, C. M., Kerton, C. R., \& Pomerleau, C. 2003, ApJS, 144, 47

Ferguson, A. M. N., Gallagher, J. S., \& Wyse, R. F. G. 1998, AJ, 116, 673

Hachisuka, K., Choi, Y. K., Reid, M. J., et al. 2015, ApJ, 800, 2

Heyer, M. H., Brunt, C., Snell, R. L., et al. 1998, APJS, 115, 241

Izumi, N. 2016, Ph.D Thesis, The University of Tokyo

Kennicutt, R. C. \& Evans, N. J. 2012, ARA $\& A$, 50, 531

Kobayashi, N., Yasui, C., Tokunaga, A. T., \& Saito, M. 2008, ApJ, 683, 178

Schruba, A., Leroy, A. K., Walter, F., et al. 2011, AJ, 142, 37

Smartt, S. J. \& Rolleston, W. R. J. 1997, ApJ (Letters), 481, L47

Snell, R. L., Carpenter, J. M., \& Heyer, M. H. 2002, ApJ, 578, 229

Strasser, S. T., Dickey, J. M., Taylor, A. R., et al. 2007, AJ, 134, 2252

Sun, Y., Xu, Y., Yang, J., et al. 2015, ApJ (Letters), 798, L27

Wolfire, M. G., McKee, C. F., Hollenbach, D., \& Tielens, A. G. G. M. 2003, ApJ, 587, 278

Wright, E. L., Eisenhardt, P. R. M., Mainzer, A. K., et al. 2010, AJ, 140, 1868 